\title{
Mechanism of reproduction effect of Adansonia digitata (Linn) root bark in female Wistar rats
}

\author{
ljeoma C Chibuogwu, Agnes I Nwannenna
}

\section{Department of Theriogenology and Production, Ahmadu Bello University, Zaria, Nigeria}

Previously, investigation had shown that Hexane-Acetyl acetate Fraction (HAAF) and Acetyl acetate-Methanol Fraction (AAMF) of Adansonia digitata (Linn) root bark extract have reproduction effect in normal cycling female Wistar rats.

Gas Chromatography and Mass Spectrometry was done to identify tcompounds present in each fraction and investigation was carried out to elucidate the mechanism of action of HAAF and AAMF on female reproduction. Thirty female rats weighing 100-120g had their estrous cycles synchronized and were randomly distributed into three groups (I, II, and III) of ten rats each. Each group received 0, 150, and $300 \mathrm{mg} / \mathrm{kg}$ body weight of each fraction per os from day of proestrus to day-seven. On day-one, day-four, day-five, day-six and day-seven, blood was collected from two rats in each group. Blood collected was analyzed for hematology, lipid profile and hormonal profile of estrogen, progesterone and follicle stimulating hormones. Uterus, ovary and vagina was processed for histopathology examination. Uteri and ovaries were also processed for immunohistochemistry study of Estrogen, Progesterone and Testosterone Receptor proteins. Fraction that regulated a receptor protein was further investigated for its effect on the gene expression of that receptor. Mechanism of action of A. digitata (L) root bark on the reproduction of female Wistar rats was then deduced from the effects of the fraction and the bioactivities of the compounds present.

HAAF and AAMF contained Oleic acid (5.57\% and $15,25 \%$ respectively); while AAMF exclusively contained Acetonotrile,2.2'-iminobis- (14.54\%); 1.alpha.,2.alpha.-Epoxy-1.beta.-methylcholesta-4,6-diene-3-one (5.40\%); 9,19Cyclolanost-23-ene-3,25-diol3-acetate,(3.beta.,23E)- (8.45\%); 8,11-Eicosadienoic acid, methyl ester (6.77\%) and 11,13Eicosadienoic acid methyl ester (8.14\%). HAAF and AAMF caused mass atresia of antral follicles at proestrus and prolonged proestrus and diestrus phases. AAMF treated rats had significantly $(\mathrm{p}<0.05)$ lower relative ovarian weights and serum estradiol levels at proestrus when compared to control rats. AAMF also upregulated ER beta proteins in endometrial glandular epithelial cells but downregulated ER alpha gene expression in endometrial stromal cells.

Its mechanism of action may involve the activities of Oleic acid which inhibits aromatase and hydroxysteroid 17 beta dehydrogenase enzyme activities in estrogen steroidogenesis. It may also involve Acetonotrile, 2.2 '-iminobis- which may act as an antagonist to androgen and estrogen-alpha signaling pathways. 